\title{
The grass is always greener on the other side
}

\section{Christoph Bosshard}

Dr. med., Vizepräsident der FMH, Mitglied des Zentralvorstandes der FMH, Departementsverantwortlicher Daten, Demographie und Qualität / Schweizerische Akademie für Qualität in der Medizin SAQM

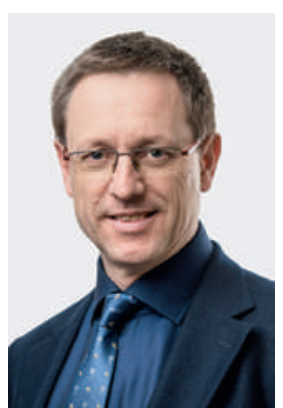

Es scheint sich um einen unserer Urinstinkte zu handeln: Wir vergleichen. In den Spiegelbildern nehmen wir uns wahr. Manchmal sagen sie uns Neues, manchmal bestätigen sie Bekanntes. Dank des internationalen «Health Policy Survey» des Commonwealth Fund, welcher 2015 zum zweiten Mal seit 2012 die Hausärztinnen und -ärzte in der Schweiz befragt hat, verfügen wir über aktuelle Erkenntnisse zur Situation der Grundversorger, welche auch untermauern, was seitens FMH in ihrer jährlichen statistischen Aufarbeitung der

\section{Phänomen Ärztedemographie:}

Die Leistungserbringer sind zunehmend auch die Leistungsbezüger.

Daten zum Teil bereits offensichtlich war: Die Dynamik der Ärztedemographie zeigt in unserem Land eine exquisite Problematik - die Leistungserbringer sind zunehmend die Leistungsbezüger. Die Alterspyramide der Grundversorger gestattet keine Hoffnung auf genügend Nachwuchs. Die entsprechenden Warnrufe ertönen aus Ärztekreisen bereits seit mehr als zehn Jahren, als sich unsere Gesellschaft bzw. die Politik noch mit der Vorstellung einer Ärzteschwemme auseinandersetzte. Die Situation der Grundversorger ähnelt auch jener aller anderen Spezialisten. Die FMH setzt sich deshalb dafür ein, dass die Schweiz ihren Ärztebedarf selber decken kann. Der aktuell notwendige Ärzte-Import stellt uns vor das moralische Dilemma des Brain-Drains und wird in Zukunft auch nicht mehr so weitergehen können. Ebenfalls gilt es, unsere Ärzte nicht durch redundante oder unnötige Bürokratie vom Patienten fernzuhalten.

Die stetige Zunahme des Frauenanteils lässt ebenfalls aufhorchen. Traditionellerweise sind Männerberufe eher gewinnorientiert, Frauenberufe hingegen sozial ausgerichtet. Dass der Arztberuf zu einem Frauenberuf geworden ist, lässt sich kaum mehr bestreiten. Mit dem Phänomen des zunehmenden Frauenanteils einher geht auch die Zunahme der Teilzeit-Tätigkeit, welche eindeutig kein alleiniges Geschlechter-Phänomen ist. Dieser Herausforderung ist ebenfalls Rechnung zu tragen, wenn wir über die zukünftige ärztliche Versorgung diskutieren.
Mit Schmunzeln habe ich die Aussagen bezüglich bester Vernetzung einerseits und niedrigster Informatisierung andererseits zur Kenntnis genommen. Natürlich kann dies mit der Altersstruktur erklärt werden. Aber dennoch wird auch klar, dass Vernetzung nicht alleine durch Strukturen und Prozesse hergestellt werden kann, sondern dass das zentrale Element das gegenseitige Kennen, das Vertrauen darstellt. Dieses Grundprinzip gilt offensichtlich sowohl für die ArztPatient-Beziehung wie auch für die Beziehungen innerhalb der Ärzteschaft. In der gleichen Ausgabe der Schweizerischen Ärztezeitung ab Seite 789 erfahren Sie mehr zur internationalen «Health Policy Survey» 2015. Unser Gesundheitswesen zeigt im internationalen Vergleich vielerorts Spitzenwerte, so etwa auch bei der raschen und freien Verfügbarkeit ärztlicher Hilfe. Mehrfach hat sich der Schweizer Stimmbürger an der Urne klar dafür ausgesprochen. Wenn nun unnötige medizinische Leistungen als Herausforderungen genannt werden, so ist dies wohl auch als Nebenwirkung des Systems zu sehen. Dass die FMH diese Thematik ernst nimmt, hat sie im Grundlagenpapier Overuse unnötige Behandlungen als Qualitätsproblem [1] zum

\section{Die Teilzeit-Tätigkeit ist kein alleiniges}

Geschlechter-Phänomen und bei der zukünftigen ärztlichen Versorgung zu berücksichtigen.

Ausdruck gebracht. Weitere Grundlagearbeiten haben auch zu Shared Decision Making und Indikationsqualität stattgefunden.

Für uns ist klar, dass wir nur gut bleiben können, wenn wir uns stetig verbessern. Aber auch hier gelten die Prinzipien von Wirksamkeit, Zweckmässigkeit und Wirtschaftlichkeit: Welchen Grenz-Nutzen leisten wir uns mit welchem Einsatz? Diese Diskussionen müssen mit einer positiven Grundhaltung geschehen. Nur so werden wir die Faszination unseres Berufes auf jüngere Generationen übertragen können, und sie zur Mitarbeit motivieren.

\section{Literatur}

1 Gerber M, Kraft E, Bosshard C. Overuse - unnötige Behandlungen als Qualitätsproblem. Schweiz Ärztezeitung 2016;97(7):236-43. 